\title{
HUELLAS DEL COREM Y LA TSD EN EL DESARROLLO DE LA DIDÁCTICA DE LA MATEMÁTICA EN ESPAÑA Y ARGENTINA ${ }^{\alpha}$
}

\author{
Footprints of COREM and TSD in the development of Didactics \\ of Mathematics in Spain and Argentina
}

\section{Pilar Orús Báguena ${ }^{\beta}$ y Dilma Fregona}

Fecha de recepción: 19/06/2019 • Fecha de aceptación: 24/07/2019

Resumen. En este trabajo intentamos mostrar aspectos del nacimiento y desarrollo de la didáctica de la matemática en Francia iniciada por uno de sus pioneros, Guy Brousseau, y la influencia de ese desarrollo en parte de las comunidades de España y Argentina. Una primera sección trata el nacimiento y desarrollo de la teoría de situaciones didácticas (TSD) a comienzos de la década de los setenta. Una institución creada en esa época, el Centre d'Observation et de Recherche pour l'Enseignement des Mathématiques (COREM) cumplió un papel clave para confrontar en la práctica de enseñanza de una escuela pública, los desarrollos teóricos que investigadores formados y en formación desarrollaron durante más de 25 años. La segunda sección muestra aspectos institucionales de ese Centro, la trama entre sus actores y los recursos producidos. Una tercera sección está destinada a una breve historia del desarrollo del campo de investigación en España y Argentina, nuestros países de origen. Por el apoyo recibido de los respectivos estados, e incluso de Francia, pudimos acceder a la formación de posgrado en el ámbito de la Universidad de Bordeaux. Finalmente, la creación en 2010 de un Centro de Recursos en

\footnotetext{
${ }^{\alpha}$ Una primera versión de este trabajo fue presentada oralmente y discutida en el IV Congreso Iberoamericano de Historia de la Educación Matemática, organizado por el Centro de Estudios sobre la Memoria Educativa de la Universidad de Murcia, realizado en Murcia del 14 al 17 de noviembre de 2017. Nuestro particular agradecimiento a las Profesoras Dolores Carrillo Gallego y Encarna Sánchez Jiménez por el constante y generoso apoyo brindado.

B Instituto de Matemáticas y sus Aplicaciones de Castelló (IMAC), Universitat Jaume I. Av. Vicent Sos Baynat, s/n 12071 Castellón de la Plana, España. orus@uji.es

₹ Facultad de Matemática, Astronomía, Física y Computación (FAMAF), Universidad Nacional de Córdoba. Av. Medina Allende s/n, Ciudad Universitaria, CP: X5000HUA, Córdoba, Argentina. fregona@famaf.unc.edu.ar
}

Cómo citar este artículo: Orús Báguena, Pilar y Dilma Fregona. «Huellas del COREM y la TSD en el desarrollo de la didáctica de la matemática en España y Argentina». Historia y Memoria de la Educación 11 (2020): 553-594 
Castellón, España, donde se albergan documentos producidos en el COREM nos estimula a estudiar e invitar a otros colegas a profundizar esa tarea. Mostramos un ejemplo de un trabajo conjunto que estamos haciendo en los últimos años.

Palabras clave: Didáctica de la matemática (DM); Teoría de situaciones didácticas (TSD); Centre d'Observation et de Recherche pour l'Enseignement des Mathématiques (COREM); CRDM-Guy Brousseau (Castellón, España).

Abstract. In this paper we try to show aspects of the birth and development of didactics in France, initiated by one of its pioneers, Guy Brousseau, and the influence that this development had certain communities of Spain and Argentina. A first section deals with the birth and development of the theory of didactical situations (TSD) at the beginning of the seventies. An institution created at that time, the Center d'Observation et de Recherche pour l'Enseignement des Mathématiques (COREM) played a key role in confronting the teaching practice of a public school with the theoretical developments that trained and in-training researchers developed for more than 25 years. The second section shows institutional aspects of that Center, the relationship among its agents and the resources produced. A third section is dedicated to a brief history of the development of the research field in Spain and Argentina, our countries of origin. Thanks to the support received from our respective states, and from France, we were able to access postgraduate training at the University of Bordeaux. Finally, the creation in 2010 of a Resource Center in Castellón, Spain, where documents produced at COREM are housed, has encouraged us to study and invite other colleagues to delve deeper into this work. We present here an example of a joint project we have been working on in recent years.

Keywords: Didactics of mathematics (DM); Theory of didactical situations in mathematics (TSD); Center d'Observation et de Recherche pour l'Enseignement des Mathématiques (COREM); Resource Center of Castellón (Spain).

\section{INTRODUCCIÓN}

La historia del campo de la Educación Matemática (EM) estuvo vinculada desde sus orígenes al campo de la educación y de la psicología, es decir en relación con las ciencias humanas.

En 1992 Kilpatrick señala los inicios del siglo XX como un período en el cual los matemáticos mostraron preocupación por la formación de miembros de su comunidad, y de cómo enseñar matemática en la 
escuela y qué matemática enseñar. ${ }^{1}$ La «juventud» de este campo científico específico, contrasta con la gran profusión de problemas afrontados.

Años más tarde, el mismo autor afirma:

La historia de la investigación en educación matemática es parte de la historia de nuestro campo -la educación matemática. Este se ha desarrollado durante los últimos dos siglos debido a que matemáticos y educadores han enfocado su atención hacia qué matemáticas se enseñan y se aprenden en la escuela y cómo se llevan a cabo estos procesos; también se han interesado en el qué y en el cómo de las matemáticas que deberían enseñarse y aprenderse en la escuela. Desde su comienzo, la investigación en educación matemática ha sido también modelada por fuerzas provenientes del campo más general de la investigación educativa, la cual abandonó, hace aproximadamente un siglo, la especulación filosófica en favor de un enfoque más científico. Al igual que la educación matemática, la investigación en este campo ha tenido que luchar para lograr su propia identidad. Ha tratado de formular su propia problemática y sus propias formas de tratarla. Ha intentado definirse a sí misma y constituir un grupo de personas que se auto identifiquen como investigadores en educación matemática. ${ }^{2}$

También Waldegg en 1998, desde la comunidad mexicana, al plantear La educación matemática ¿una disciplina científica? ${ }^{3}$ expuso muchos de los enfoques incluso contradictorios y de las cuestiones e investigaciones que en esa década se plantearon sobre la EM desde diversas comunidades científicas.

En la actualidad, la EM es un campo en movimiento y transformación, y como en todo campo académico se manifiestan allí tensiones

\footnotetext{
1 Jeremy Kilpatrick, «A History of Research in Mathematics Education», en Handbook for Research on Mathematics Teaching and Learning, ed. Douglas Grouws (New York: Macmillan, 1992), 3-38.

2 Jeremy Kilpatrick, «La investigación en educación matemática: su historia y algunos temas de actualidad», en Educación Matemática, eds. Jeremy Kilpatrick, Pedro Gómez y Luis Rico, 1-18. (Bogotá: Una empresa docente \& Grupo Editorial Iberoamérica, 1995), 1.

${ }^{3}$ Guillermina Waldegg, https://biblat.unam.mx/es/revista/coleccion-pedagogica-universitaria/articulo/la-educacion-matematica-una-disciplina-cientifica (consultado el 10-01-2019).
} 
entre disciplinas y enfoques teóricos. En 2018, en conmemoración de los treinta años de publicación ininterrumpida de Educación Matemática, la primera revista de habla hispana dedicada a esta temática, se editó en México un libro coordinado por Alicia Ávila. Hemos seleccionado algunos párrafos de la introducción, donde se señalan aspectos del estado actual de la EM en Iberoamérica, relativos a la investigación y la historia. El subrayado es nuestro.

Se trata de un libro de celebración, de festejo por los 30 años de publicación ininterrumpida de Educación Matemática, importante vehículo de comunicación de la investigación en educación matemática que se difunde en habla hispana. [...]

La solicitud planteada [a los autores] tenía como objetivo Mostrar los inicios y el desarrollo de la educación matemática en Iberoamérica desde la perspectiva actual de los constructores del campo. En función de tal objetivo, se les pidió discutir algún artículo de su propia autoría publicado en Educación Matemática entre 1989 y 2003. [...]

Es cierto que delimitar geográficamente las fronteras de la investigación es inexacto, puesto que algunos de los autores hoy forman parte de la comunidad anglo-sajona o la francófona, ya sea por su lugar de residencia, o por la relevancia que en esas comunidades han alcanzado sus trabajos. Sin embargo, con tal delimitación se quiere enfatizar la existencia de una comunidad de habla hispana interesada en la educación matemática desde las últimas décadas del siglo XX y a la cual debemos aportes importantes para la comprensión de los fenómenos de la enseñanza y el aprendizaje de las matemáticas en nuestro contexto. ${ }^{4}$

Y más adelante,

Muchos de los autores de este libro han contribuido a diseñar propuestas educativas en matemáticas. Pero la investigación ha ido más allá y se han desarrollado rutas que analizan las formas

\footnotetext{
${ }^{4}$ Alicia Ávila, Rutas de la educación matemática: 30 años de investigación en la revista Educación Matemática (México: Sociedad Mexicana de Investigación y Divulgación de la Educación Matemática A. C. (SOMIDEM), 2018), 7-8.
} 
que han tomado en la práctica reformas curriculares de gran escala nacidas con el fundamento de la investigación en didáctica (Block) o que, a falta de ella (Ávila), impulsaron la indagación sobre los aspectos implicados en la innovación y su puesta en marcha. ${ }^{5}$

Ávila plantea además la pluralidad como una condición del estado del arte en el campo y como problemática en la organización del libro.

Cómo organizar la pluralidad no es pregunta nueva en el campo de la educación matemática: es una cuestión que ha provocado diferentes reacciones y respuestas por parte de investigadores de este campo. Es también una pregunta que hube de enfrentar para organizar y dar estructura al libro. [...]

Ahora bien, es de mi interés señalar la dificultad para organizar las partes constitutivas del libro conforme a objetivos, marcos teóricos o metodologías debido a la diversidad de rutas de indagación delineadas en los textos..

En México conviven dos modos de designar el campo de investigación: «Matemática Educativa»y, como lo evidencian las publicaciones citadas, «Educación Matemática». Esta última expresión es ampliamente utilizada en países anglosajones y latinoamericanos como Brasil, e inclusive en instituciones como el ICMI (International Commission on Mathematical Instruction). En Argentina conviven esa designación y también, por la influencia francesa, «Didáctica de la Matemática»(DM). En este artículo, por nuestra trayectoria de formación, utilizamos DM.

En Fregona y Orús:

Bajo las denominaciones «Didáctica de la Matemática»o «Educación Matemática» existen diversas acepciones. En el lenguaje habitual, se las identifica con la enseñanza de la matemática, aunque también se las reconoce como área de investigación en la cual la enseñanza de las matemáticas es uno de sus objetos

\footnotetext{
5 Ávila, Rutas de la educación matemática, 11.

6 Ávila, Rutas de la educación matemática, 14.
} 
de estudio, entre otras problemáticas abordadas desde diversas perspectivas teóricas. ${ }^{7}$

Las mesas de trabajo en los últimos congresos internacionales ${ }^{8}$ dan una idea de la diversidad de los temas de estudio, muchos de ellos comunes aunque pertenezcan a diferentes comunidades y perspectivas teóricas. Como lo anticipamos en el título, abordaremos aspectos de la denominada "escuela francesa de didáctica de la matemática», cuyos pioneros son Guy Brousseau y Gérard Vergnaud.

\section{APUNTES SOBRE LA HISTORIA DE LA DM EN FRANCIA}

Algunas publicaciones producidas con la intención de historiar la construcción del campo de la DM-EM en el mundo occidental, y en Francia en particular, señalan como evento de importancia considerable el lanzamiento por los soviéticos del primer Sputnik en el año 1957. ${ }^{9} \mathrm{El}$ miedo a quedar atrasados científicamente sirvió de estímulo a diferentes países para el desarrollo de nuevos programas de matemáticas, así como a inversiones en el desarrollo de comunidades de investigación en matemática, física, biología, astronomía, etc. En Francia, en 1967, se crea una comisión ministerial para la renovación de la enseñanza presidida por Lichnerowicz. Se declaró explícitamente la finalidad democrática de la reforma y los programas incluyeron nuevos temas en la escolaridad secundaria tales como la teoría elemental de conjuntos, estructuras algebraicas y nociones de cálculo diferencial e integral y se suprimió la geometría euclidiana tradicional. Dicha reforma curricular se conoce como «reforma de la matemática moderna» y para hacerla efectiva en las aulas, a partir de 1969 el Ministerio de Educación Nacional de Francia creó, asociados a universidades, los IREMs. Así se denomina a los Instituts de Recherche pour l'Enseignement des Mathématiques, donde trabajaban docentes de escuelas primaria y secundaria que enseñaban

\footnotetext{
${ }^{7}$ Dilma Fregona y Pilar Orús, La noción de medio en la teoría de las situaciones didácticas. Una herramienta para analizar decisiones en las clases de matemática (Buenos Aires: Libros del Zorzal, 2011), 15.

${ }^{8}$ Véase por ejemplo: http://www.seiem.es/; http://igpme.org/ ;http://www.mathunion.org/icmi/; http://www.icme13.org/discussion_groups; http://www.icme13.org/survey_teams (consultado el 2211-2018).

9 Jesús Hernández, La enseñanza de las matemáticas modernas (Madrid: Alianza Editorial, 1978), 14.
} 
matemática, matemáticos, inspectores, psicólogos, sociólogos, lingüistas, etc. A pesar de su denominación estos institutos tuvieron en su origen esencialmente una vocación de formación: se trataba de estudiar con los docentes los contenidos de los nuevos programas, y también de promover la innovación pedagógica en el marco de dicha reforma. En 1969, por gestión del Prof. Jean Colmez, se creó en Bordeaux uno de esos Institutos y fue esa institución, como en otras universidades, la que albergó la DM hasta el momento en que pudo ser reconocida en las estructuras usuales de la investigación. Como afirma Rouchier «La didactique des mathématiques est un fragment de l'histoire des IREM».10

En junio de 1993, se realizó en París un coloquio en homenaje a Brousseau y Vergnaud denominado: "Vingt ans de didactique des mathématiques en France». De este modo se reconocían los trabajos y propuestas teóricas difundidas en el transcurso de los años setenta, que resumen y representan la ruptura que se produce en esa época. Rouchier ${ }^{11}$ destaca dos artículos de Brousseau y uno de Vergnaud donde se establece una parte esencial de los fundamentos de la didáctica. El primer texto de Brousseau, Processus de mathématisation, fue publicado en 1972 por la Association des Professeurs de Mathématiques de l'Enseignement Publique. El segundo texto Peut-on améliorer le calcul des produits des nombres naturels?, difundido en 1973 por el IREM de Bordeaux. Vergnaud, en colaboración con Catherine Durand, publica en 1976 «Structures additives et complexité psychogénétique» en la Revue Française de Pédagogie.

Organizado por la Association pour la Recherche en Didactique des Mathématiques (ARDM), la publicación surgida de ese evento presenta un balance de veinte años de trabajo que hicieron un lugar a la DM como campo de investigación organizado sobre la difusión de las matemáticas en las sociedades, en particular a través de la enseñanza.

En la década de los setenta muy pronto otros investigadores contribuyeron al desarrollo de nuevas perspectivas teóricas y profundizaciones, entre otros Régine Douady, Yves Chevallard, Michèle Artigue, Régis

\footnotetext{
10 André Rouchier, «Naissance et développement de la didactique des mathématiques», en Vingts ans de didactique des mathématiques en France, eds. Michèle Artigue, Régis Gras, Colette Laborde, Patricia Tavignot (Grenoble: La Pensée Sauvage éditions, 1994), 153.

11 Rouchier, «Naissance et développement», 149-150.
} 
Gras, Colette Laborde, Nicolas Balacheff. Las concepciones de la DM que se fueron consolidando, supusieron desde su origen una ruptura epistemológica respecto a los paradigmas investigativos de la época y fueron aportando diferentes dimensiones a la problemática de la comunicación de saberes matemáticos.

Desde 1980, la ARDM organiza cada dos años la Escuela de Verano, un espacio destinado a compartir y difundir investigaciones, debatir problemáticas desde diferentes perspectivas teóricas, que cuenta con la participación de investigadores formados y en formación de distintos países. En ese primer encuentro, Yves Chevallard presentó en un curso las notas preparatorias de La Transposition Didactique. ${ }^{12}$ El libro vio la luz en 1985, y luego una versión ampliada se dio a conocer en 1991.

En el año 2003 Brousseau recibió la primera medalla Félix Klein, otorgada por la International Commission on Mathematical Instruction (ICMI) por su destacada contribución a la EM, ${ }^{13}$ creando y desarrollando la teoría de las situaciones didácticas (TSD).

En el siguiente apartado, nos interesa mostrar aspectos del desarrollo de la TSD y de ciertas condiciones que consolidaron esa perspectiva.

\section{La TSD: nacimiento y desarrollo}

Desde los comienzos de la década de los sesenta Brousseau, matemático y educador, comenzó a formular proyectos en torno a mejorar la enseñanza de la matemática desde una perspectiva científica. ${ }^{14}$ Como ya dijimos, los primeros conceptos los hace públicos en la década de los setenta y plantean una ruptura con investigaciones occidentales de la época. Por un lado, distingue la DM y los aportes provenientes de otras disciplinas, en particular la psicología y la pedagogía, con la plena

\footnotetext{
12 Yves Chevallard, La transposition didactique. Du savoir savant au savoir enseigné. Deuxième édition (Grenoble: La Pensée Sauvage 1991/1985).; Yves Chevallard, La transposición didáctica. Del saber sabio al saber enseñado, ed. en español (Buenos Aires: Aique, 1997).

${ }_{13}$ El ICMI, además de la Medalla Félix Klein otorga también la Medalla Hans Freudenthal. La primera se concedió en 2003 a Celia Hoyles. Entre otros investigadores galardonados mencionamos a Ubiratan D'Ambrosio (2005), Jeremy Kilpatrick y Anna Sfard (2007) e Yves Chevallard (2009).

14 Marie-Jeanne Perrin-Glorian, «Théorie des situations didactiques: naissance, développement, perspectives», en Vingts ans de didactique des mathématiques en France, eds. Michèle Artigue, Régis Gras, Colette Laborde, Patricia Tavignot (Grenoble: La Pensée Sauvage éditions, 1994), 97-147, 98.
} 
vigencia de los trabajos de Piaget, Greco, Dienes, Gattegno, etc. Por otro, introdujo los saberes (matemáticos en particular) como componente determinante de las situaciones didácticas. Esto contribuyó a superar el clásico binomio alumno-profesor de las aproximaciones de naturaleza pedagógica y generalista.

Una parte fundamental del proyecto de estudio sobre la enseñanza de la matemática desde una perspectiva científica era crear un centro que favoreciera una interacción apropiada entre investigadores en DM y un establecimiento del sistema educativo público donde fuera posible observar a alumnos y maestros en condiciones propicias.

En el año lectivo 1972-73 se crea el Grupo Escolar Jules Michelet en Talence, un municipio próximo a Bordeaux. Ya estaba entonces una de las instituciones, una escuela pública. Poco después, en 1974, con el apoyo de académicos de la Universidad y el IREM de Bordeaux, y de autoridades del sistema educativo, Brousseau creó en ese Grupo Escolar el Centre d'Observation et de Recherche pour l'Enseignement des Mathématiques, (COREM). Con aportes del Estado, se construyó el edificio que albergó al Centro hasta 1999. Más información sobre esta institución, véase el sitio de Brousseau. ${ }^{15}$ Volveremos sobre aspectos de la organización y del funcionamiento de este Centro.

En la Segunda Escuela de Verano de Didáctica de la Matemática en 1982, Brousseau presentó una nueva noción de la TSD, la de ingeniería didáctica. Allí precisa el significado del término situación en el modelo teórico:

Une situation didactique est un ensemble de rapports établis explicitement et/ou implicitement entre un élève ou un groupe d'élèves, un certain milieu (comprenant éventuellement des instruments ou des objets) et un système éducatif (le professeur) aux fins de faire approprier à ces élèves un savoir constitué ou en voie de constitution. ${ }^{16}$

\footnotetext{
15 Guy Brousseau, «Présentation du COREM», 2015. http:/guy-brousseau.com/le-corem/presentation/ (consultado el 29 -11-2018).

${ }^{16}$ Guy Brousseau, «L'ingénierie didactique. D'un problème à l'étude a priori d'une situation didactique», en Actes de la 2ème école Ecole d'été de didactique des mathématiques (Olivet 1982, IREM d'Orléans), 39. http://guy-brousseau.com/wp-content/uploads/2012/03/82-3-Dun-probl\%C3\%A8me$\% \mathrm{C} 3 \% \mathrm{~A} 0-1 \% \mathrm{C} 3 \% \mathrm{~A} 9$ tude-a-priori.pdf (consultado el 22-11-2018).
} 
Años después, en una publicación gris del IREM de Bordeaux, Brousseau enfatiza la intencionalidad de la enseñanza en el marco de un proyecto social, y en esas condiciones, «la didactique devient le projet social de faire approprier -par un ou des élèves- un savoir constitué ou en voie de constitution». ${ }^{17} \mathrm{El}$ acto de enseñar es interpretado así como el ejercicio mismo de la didáctica.

En 1989, al discutir distintas acepciones de la palabra didáctica, caracteriza el campo de investigación en el cual trabaja del siguiente modo:

Desde hace una quincena de años ha aparecido también bajo el nombre de «didáctica», un intento de constituir una ciencia de la comunicación de los conocimientos y de sus transformaciones; una epistemología experimental que intenta teorizar la producción y la circulación de los saberes un poco como la economía estudia la producción y la distribución de los bienes materiales. Esta ciencia se interesa en lo que estos fenómenos tienen de específico del conocimiento que se tiene en el punto de mira. ${ }^{18}$

En este artículo, aparece la expresión epistemología experimental. Más recientemente, el autor retoma esa terminología de los años setenta y justifica ciertas decisiones con respecto a la denominación del campo:

[«Epistémologie expérimentale des mathématiques»] c'était une bonne dénomination pour notre travail sur les situations et les curriculums. Toutefois, nous l'avons rejetée: d'abord pour nous rapprocher des professeurs de mathématiques à qui nos IREM se consacraient et à qui notre «Science» devait s'adresser finalement, ensuite pour de ne pas trop nous démarquer des autres DEA en cours de création dans les IREMs. La «Didactique des Mathématiques» serait pour les professeurs ce qu'est «la Science Médicale» pour les médecins, et l'épistémologie

\footnotetext{
17 Guy Brousseau, «La tour de Babel», Études en Didactique de Mathématiques, Article occasionnel 2. Bordeaux: IREM de Bordeaux (1988), 1.

18 Guy Brousseau, «Utilité et intérêt de la didactique pour un professeur de collège», Petit X 21 (1989): 48-68. Guy Brousseau, «¿Qué pueden aportar a los enseñantes los diferentes enfoques de la didáctica de las matemáticas? Primera parte», Enseñanza de las Ciencias 8, no.3 (1990): 260.
} 
expérimentale des mathématiques serait sa Biologie. Nous avons rejeté «Méthodologie» qui existait déjà et aussi «Didactologie», logique mais orienté vers l'intelligence artificielle, et à cause de leur résonance normative commune. Nous préférions «Observation» qui à l'époque semblait aller de soi. ${ }^{19}$

Estas referencias, de distintas épocas, muestran las huellas en la construcción de la TSD. En esta última cita están claramente identificadas la Didáctica de la Matemática, como campo de investigación, y la Observación en la construcción de la teoría.

Documentos de los años setenta dan cuenta del esfuerzo por precisar métodos de investigación ${ }^{20}$ a través de numerosas publicaciones del IREM de Bordeaux y un artículo sobre la observación en la Revue Française de Pédagogie (1978). Allí, el vínculo entre la observación y la construcción de la teoría es explícito: «L'utilisation d'une observation exige toujours un effort pour discerner ce qui était possible dans ce qui ne s'est pas produit et ce qui est nécessaire dans ce qui s'est produit». ${ }^{21}$

Años más tarde, Brousseau describe su perspectiva con respecto a la psicología cognitiva:

En los años 60, con algunos años de experiencia como maestro de escuela primaria, y siendo estudiante de matemática un profesor me mandó a estudiar psicología cognitiva con Pierre Greco. Me impresionó Greco por su habilidad para concebir dispositivos experimentales destinados a poner en evidencia la originalidad del pensamiento matemático de los niños en las etapas de su desarrollo. Sin embargo, me daba cuenta que no entraba entre sus preocupaciones analizar los dispositivos mismos y hacer explícita la relación entre éstos y la noción matemática cuya adquisición era estudiada. Comencé a plantearme algunas cuestiones: ¿En qué condiciones puede propiciarse que un sujeto cualquiera

\footnotetext{
19 Guy Brousseau, «1975: épistémologie expérimentale vs Didactique 2016». https://guy-brousseau. com/3297/1975-epistemologie-experimentale-vs-didactique-2016/ (consultado el 22-11-2018).

${ }^{20}$ Perrin-Glorian, «Théorie des situations didactiques», 106.

${ }^{21}$ Guy Brousseau, «L'observation des activités didactiques», Revue Française de Pédagogie 45 (1978): 134. hal-00515106.
} 
tenga la necesidad de un conocimiento matemático determinado para tomar ciertas decisiones?, y ¿cómo explicar de antemano la razón por la cual lo haría?.22

La TSD propuso una construcción que permite comprender las interacciones sociales entre alumnos, docentes y saberes matemáticos que se dan en una clase y condicionan lo que los alumnos aprenden y cómo lo aprenden. Esta construcción fue un trabajo colectivo del cual participaron investigadores, estudiantes de grado y postgrado, docentes y también alumnos de diferentes niveles de escolaridad.

Gascón analizó la evolución de la problemática didáctica y distingue el momento de la aparición de la modelización de la TSD:

El nuevo punto de vista en didáctica de las matemáticas, la didáctica fundamental, nació precisamente cuando Brousseau vislumbró por primera vez la necesidad para la didáctica de utilizar un modelo propio de la actividad matemática, dado que los modelos epistemológicos usuales no habían sido construidos para responder a los mismos problemas que se plantea la didáctica. Históricamente se corresponde con las primeras formulaciones de la teoría de las situaciones didácticas, cuyo texto fundador fue publicado a principios de los años 70. En esto consiste, precisamente, el principio metodológico fundamental de la teoría de las situaciones: definir un "conocimiento matemático» mediante una «situación», esto es, por un autómata que modeliza los problemas que únicamente este conocimiento permite resolver de forma óptima (Brousseau, 1994).23

\section{La observación y el COREM}

Tan importante para el desarrollo de la DM es el marco teórico propuesto por la TSD como la metodología que acompañó esta

\footnotetext{
22 Guy Brousseau, Iniciación al estudio de la teoría de las situaciones didácticas (Buenos Aires: Libros del Zorzal, 2007), 14-15.

${ }_{23}$ Josep Gascón, «Evolución de la didáctica de las matemáticas como disciplina científica», Recherches en Didactique des Mathématiques 52 (1998): 7-33, 18. http://2633518-0.web-hosting.es/blog/didact_mate $/ 2$.Evoluci $\%$ C3\%B3n $\% 20 \mathrm{de} \% 20 \mathrm{la} \% 20 \mathrm{did} \% \mathrm{C} 3 \% \mathrm{~A} 1 \mathrm{ctica} \% 20 \mathrm{de} \% 20 \mathrm{las} \% 20 \mathrm{matem} \% \mathrm{C} \% \mathrm{~A}$ 1ticas.pdf, (consultado el 22-11-2018).
} 
construcción teórica desde sus inicios, en particular la observación de las actividades didácticas. El COREM no fue solamente un edificio sino un entramado colaborativo de investigación en torno a las escuelas públicas Michelet de Talence.

Desde la perspectiva de la TSD los investigadores y en consecuencia también los observadores no interactuaban con los alumnos en el momento de la clase (eventualmente se realizaban entrevistas en otros espacios para analizar un caso), sólo observaban; ya que el objeto de estudio son las situaciones en sí mismas, la manera en que nos informan sobre los conocimientos y los saberes que ponen en juego y que movilizan los diferentes actores de la relación didáctica. Así, no se estudia al actor en abstracto, sino en la situación, en la potencialidad que puede ofrecer al alumno, para desarrollar su actividad matemática y al maestro como actor en esa institución didáctica.

La DM como dominio científico y como la entendía Brousseau, planteó desde sus inicios métodos específicos de investigación, entre ellos la Observación y lo que él llama «método espiral» de investigación. La Observación, con mayúscula, es para Brousseau la metodología global de experimentación para poder confrontar con la contingencia los diferentes conceptos y modelos teóricos de las matemáticas en situación escolar. Esta metodología incluye varios instrumentos metodológicos, entre ellos, las fichas didácticas, la observación de clases propiamente dicha, la reacción «à chaud» (en «caliente») del enseñante tras la lección, las mesas redondas y debates post-observación, registros cuantitativos, etc. ${ }^{24}$

\footnotetext{
${ }^{24}$ Groupe de Recherche sur l'Enseignement Élémentaire, Les Observations et les Tables Rondes, Compte rendu du colloque organisé par l'IREM de Bordeaux. Talence: IREM de Bordeaux, 1975, Collection «L'analyse de la didactique des mathématiques».
} 


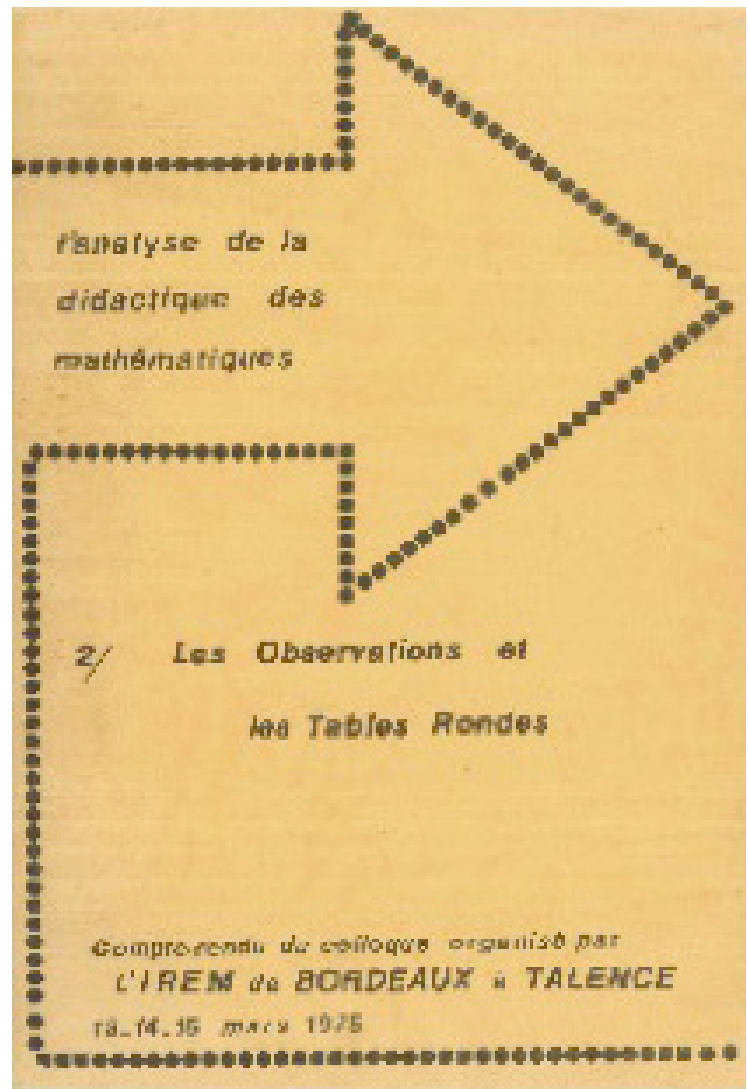

Figura 1. Carátula del monográfico sobre la Observación en el COREM, publicado por el IREM (1975).

Para que la observación no fuera «banal», se necesitaba la ficha didáctica, es decir la planificación de la clase elaborada en el marco de una investigación o con el apoyo de profesores formadores de maestros que integraban el COREM. Los docentes a cargo de los cursos donde se realizaba la observación participaban activamente en la preparación de esas lecciones, así analizaban los objetivos de la sesión y anticipaban aspectos de la gestión de la clase. También los observadores tenían acceso previamente a esas fichas didácticas, para orientarse en el transcurso de la observación. Dichas fichas se diseñaban y se analizaban teniendo en cuenta los conceptos que la modelización de la teoría (la TSD) proporciona en el marco de la llamada «ingeniería didáctica». 
Toutes ces notions [variables de commande, variables didactiques] sont reprises et développées dans les textes préparés pour l' E.E. 82, oú une partie de la réflexion se centre autour de l'ingénierie didactique qui donne lieu à deux textes: un de G. B. et un de Y. Chevallard. Le double aspect de l'ingénierie didactique comme méthodologie de recherche (G.B. employait depuis longtemps pour cela le terme de phénoménotechnique) et comme production de situations d'enseignement est souligné et le deuxième aspect distingué de l'innovation. On y aborde aussi les problèmes de reproductibilité et d'obsolescence. ${ }^{25}$

Cada observación de una determinada ingeniería, pone a prueba no solamente la situación diseñada, sino también las nociones teóricas que las sustentan; es parte del «método espiral», ya que la observación que se puede realizar gracias a la modelización de la teoría, también sirve para identificar e introducir las modificaciones pertinentes (tanto de la teoría como de su experimentación), con su reflejo en las ingenierías. En este sentido, como ya lo hemos visto en la cita anterior, algunos autores identifican la ingeniería didáctica como una metodología en sí misma. ${ }^{26}$

Este método espiral de observación, fue posible debido a la creación y existencia del COREM. Durante más de veinte años se recogieron diferentes tipos de datos como resultado del trabajo realizado en el marco de la TSD, tanto en la preparación de las lecciones como en las observaciones sistemáticas llevadas a cabo. Hay un número importante de publicaciones diversas, volveremos sobre ello.

Para profundizar lo que supuso el COREM para la TSD, así como para conocer más datos históricos sobre su concepción y desarrollo, sugerimos una visita a la web personal de Brousseau en particular la «pestaña» sobre el COREM. ${ }^{27}$

\footnotetext{
25 Perrin-Glorian, «Théorie des situations didactiques», 122.

${ }_{26}$ Michèle Artigue, «Ingénierie didactique», Recherches en didactique des mathématiques 9, no. 3 (1990): 281-308. Michèle Artigue, "Ingeniería didáctica», en Ingeniería didáctica en educación matemática. Un esquema para la investigación y la innovación en la enseñanza y el aprendizaje de las matemáticas, eds. Michèle Artigue, Régine Douady, Luis Moreno y Pedro Gómez Guzmán (Bogotá: Una Empresa Docente y Grupo Editorial Iberoamérica, 1995), 33-59.

${ }^{27}$ Guy Brousseau, «Présentation du COREM», 2015.
} 


\section{UNA EXPLORACIÓN DEL COREM}

Hemos seleccionado a continuación algunos datos y aspectos que creemos relevantes, para comprender la organización y funcionamiento del COREM e identificar recursos allí documentados.

\section{Aspectos institucionales del Grupo Escolar Michelet y el COREM28}

El Grupo Escolar Jules Michelet se creó en el año escolar 1972-73 en Talence, un municipio próximo a Bordeaux en una zona donde hay gran población de emigrantes. El Grupo cuenta con: una escuela maternal con 4 clases (grupos) de alumnos de 3, 4 y 5 años, designadas "pequeña sección», "sección media» y "gran sección» y una escuela primaria con 10 clases, que abarca alumnos desde los 6 a los 11 años. Este nivel incluye: curso preparatorio, curso elemental nivel 1, curso elemental nivel 2, curso medio nivel 1 y curso medio nivel 2. Adoptamos de aquí en adelante, la denotación del Ministerio de Educación Nacional de Francia, para los diferentes niveles: MAT Ps, MAT Ms, MAT Gs, para la maternal y CP, $\mathrm{CE} 1, \mathrm{CE} 2, \mathrm{CM} 1$ y CM2, para la primaria.

El terreno empírico contaba con un edificio acondicionado para la observación directa y el registro en video de clases de matemáticas que, curricularmente, respondían a las exigencias del sistema educativo y del IREM.

El COREM, ha sido durante sus 28 años de funcionamiento (19721999), un espacio donde era posible diseñar y llevar a cabo lecciones, observar a docentes y alumnos en sus interacciones en clase y desplegar estudios realizados por el trabajo conjunto de personas vinculadas al IREM de Bordeaux. Específicamente, investigadores y estudiantes de los postgrados en DM de la Universidad de Bordeaux-I, docentes de la escuela Michelet y profesores formadores de docentes que trabajaban regularmente con los maestros de la Escuela. Las condiciones de creación propiciaron que el COREM haya sido un lugar privilegiado para realizar

\footnotetext{
${ }^{28}$ Para más información, véase Dilma Fregona y Pilar Orús, «El Centro de Recursos en Didáctica de la Matemática Guy Brousseau: un sitio para explorar prácticas de enseñanza de las matemáticas», en Formación de profesores que enseñan matemática y prácticas educativas en diferentes escenarios. Aportes para la Educación Matemática, eds. Dilma Fregona, Silvina Smith, Mónica Villarreal y Fernanda Viola (Córdoba, Argentina: FAMAF y Universidad Nacional de Córdoba: 2017), 109-132.
} 
observaciones, no sólo por sus condiciones físicas sino también porque la participación de los investigadores formados y en formación estaban institucionalmente reguladas, las obligaciones recíprocas de unos y otros explicitadas, independientemente de las personas, lo que garantizaba un «contrato de investigación» satisfactorio.

La Escuela Michelet no era (ni es) una escuela experimental, ya que por fuera de las fases de observación, no se ponen a prueba métodos, técnicas o nuevos programas; no era una escuela piloto o modelo donde se practique una enseñanza mejor; no era un anexo o escuela de aplicación para la formación inicial de maestros; ni una escuela testigo. Era una escuela que pudiese servir para la observación de los fenómenos que la enseñanza de las matemáticas produce en las condiciones habituales de una escuela pública, en una zona suburbana periférica de la ciudad de Bordeaux.

\section{Tramas entre los actores del COREM}

Los actores en ese entramado institucional eran docentes, alumnos, formadores de docentes asignados a cada nivel de la escuela e investigadores en DM, formados y en formación. Como otros estudiantes de DM de la Universidad de Bordeaux, nosotras participamos activamente en la vida de esa comunidad educativa y de investigación durante el período en el que realizamos nuestras respectivas tesis.

Los docentes de la escuela Michelet incorporaban algunas actividades particulares a su tarea como enseñantes. Para ello su carga lectiva era reducida en unas horas semanales (18 horas lectivas sobre las 24 horas habituales) para que pudiesen participar en ciertos aspectos de la investigación, fundamentalmente:

- En la preparación de clases: tanto en la preparación de clases «comunes» con profesores formadores de maestros y eventualmente investigadores, como en la preparación de clases que eran objeto de tesis (con los respectivos doctorandos e investigadores y los profesores formadores de maestros).

- En las observaciones de clases: durante todos esos años, al menos una vez por semana, se preparaba una situación de enseñanza de 
matemática, la realizaba uno de los docentes a cargo de una de las clases. La sesión era filmada y observada y después comentada colectivamente por los miembros del COREM, entre ellos, los docentes de la escuela Michelet que estaban de observadores. Estas observaciones se realizaban semanalmente, en caso de las clases comunes o se observaba una secuencia, en el momento que era necesario, en caso de una investigación, pero siempre contando con el acuerdo de los PEN (Professeur de l'École Normale) que asesoraban a los docentes de cada nivel, para garantizar el correcto desarrollo curricular, fundamentalmente en lo relativo a la posible incidencia de las investigaciones. Es una de las precauciones deontológicas contempladas a priori por Brousseau ${ }^{29}$ en el diseño del proyecto del COREM (1965-1970).

- Participación en los seminarios semanales de investigación y de formación continua en DM (de hora y media de duración). En ellos, se analizaba la observación de clases; por un lado las observaciones destinadas a extraer y explicar fenómenos de didáctica, concernientes a la enseñanza «tal y como se practica» y por otro, las observaciones que constituyen un momento esencial de las investigaciones que utilizaban la ingeniería didáctica como metodología. ${ }^{30}$

- La redacción de las planificaciones semanales de docencia y de investigación y de los informes (Bilan) de las acciones realizadas a lo largo del curso y del desarrollo de las programaciones anuales.

La reducción de horas lectivas de los maestros, tenía además un efecto organizativo, ya que para las dos aulas que había por nivel, se necesitaban tres maestros para cubrir las 48 horas semanales de docencia, lo que permitía la disponibilidad de un profesor por nivel para realizar todas las tareas anteriormente citadas, sin desatender la docencia. A su vez exigía un esfuerzo de coordinación y trabajo colaborativo entre los

\footnotetext{
29 Disponible en la web de GB. http://guy-brousseau.com/le-corem/le-crem-projet-de-lirem-de-bordeaux-et-du-futur-corem-1967/ (consultado el 25-11-2018).

30 Para profundizar en las relaciones enseñantes investigadores del COREM, ver Marie-Hélène Salin y Denise Greslard-Nédélec, «La collaboration entre chercheurs et enseignants dans un dispositif original d'observation de classes».http://guy-brousseau.com/wp-content/uploads/2010/08/Collaboration-entre-chercheurs-et-enseignants.pdf (consultado el 29-11-2018).
} 
tres docentes, para desarrollar las lecciones, pues no tenían aula asignada: cada uno debía poder retomar la lección en una clase en donde otro de sus compañeros la había empezado. Es decir, no había un profesor $\mathrm{X}$ para el grupo A de $C M 1$ (equivalente a $4^{\circ}$ de primaria) y otro profesor $\mathrm{Y}$, para el grupo $\mathrm{B}$ del mismo nivel, quedando un tercer profesor $\mathrm{Z}$ de apoyo; sino que los tres maestros $\mathrm{X}, \mathrm{Y}$ y Z, eran profesores de los grupos A y B, de CM1. Este aspecto también se contempló en el proyecto inicial del COREM.

Este complejo entramado y la eficacia de su funcionamiento fue producto de la voluntad de los pioneros del proyecto, de que funcione de tal manera que la finalidad pedagógica de la escuela no fuese alterada por la existencia de las investigaciones, ni de las observaciones y que tanto unas como otras pudiesen desarrollarse en las mejores condiciones metodológicas posibles. Fueron 5 años (1965-1970) de preparación y de negociación de objetivos comunes en torno a la observación como lo señala Brousseau ${ }^{31}$.

\section{Los recursos producidos}

La existencia de un centro de observación como el COREM, con las características descritas en los parágrafos anteriores, ha permitido generar una gran cantidad de recursos de diversa naturaleza:

L'existence du centre permet la constitution de deux sources de données :

a) le recueil d'informations qualitatives et quantitatives sur l'enseignement des mathématiques à l'école primaire sur une longue durée: progressions suivies, fiches de préparation des séquences, travaux des élèves, épreuves d'évaluation et résultats (bruts et pour certains ayant subi une certaine analyse). Il serait possible, par exemple, de reconstituer le parcours mathématique d'un élève depuis l'école maternelle.

b) l'observation de classes avec deux modalités :

\footnotetext{
${ }_{31}$ Ver las diaporamas de la web de GB http://guy-brousseau.com/le-corem/presentation/
} 
- d'une part, des observations destinées à dégager et expliquer des phénomènes de didactique, concernant l'enseignement «tel qu'il se pratique».

- d'autre part, des observations qui constituent un moment essentiel des recherches utilisant l'ingénierie didactique comme méthodologie. ${ }^{32}$

En definitiva, queremos señalar que el funcionamiento de ese espacio de investigación teórica y de observación de prácticas de enseñanza, es el que ha favorecido las condiciones para la producción de una gran cantidad de documentos. En su web personal, Guy Brousseau reflexiona en 2013, sobre la importancia de estos recursos en sus orígenes y en la actualidad, a través de una batería de preguntas y respuestas en su artículo «Questions au sujet des documents du COREM».33 Aquí sólo hemos dado unas pinceladas.

En la actualidad las condiciones de consulta de las producciones en el marco del COREM, están explicadas por el propio Brousseau en su web, así como sus limitaciones. ${ }^{34}$ Principalmente la diferenciación entre documentos visuales y documentos papel y en consecuencia la existencia de dos instituciones de referencia para su consulta: VISA para los videos y el CRDM para los documentos en soporte papel:

Ces restrictions et diverses considérations techniques nous ont conduits à dissocier la consultation des documents visuels et celles des documents sur papier. Les premiers sont déposés auprès de VISA, ils ne sont consultables que par l'intermédiaire de la toile. Les seconds ne sont pour l'instant consultables que sur place à l’Université Jaume I de Castellon (Espagne), mais leur numérisation est en cours, et la consultation par l'intermédiaire de la toile est en cours d'organisatio. Les deux organismes coopèrent

\footnotetext{
32 Marie-Hélène Salin y Denise Greslard-Nédélec, «La collaboration entre chercheurs et enseignants dans un dispositif original d'observation de classes». http://guy-brousseau.com/wp-content/ uploads/2010/08/Collaboration-entre-chercheurs-et-enseignants.pdf, (consultado el 29-11-2018).

${ }_{33}$ En https://docs.google.com/document/d/17T8Btq7XJceCBmaPRRMw83BUCD7bZZuLEl86pdy5-9o/ edit. (consultado el 29-11-2018)

${ }^{34}$ Guy Brousseau, «Accès aux DONNEES DU COREM», 2015. http://guy-brousseau.com/le-corem/ acces-aux-documents-issus-des-observations-du-corem-1973-1999/ (consultado el 29-11-2018)
} 
parfaitement pour assurer la consultation des indispensables associations de documents. ${ }^{35}$

Los cerca de 450 videos que han podido ser recuperados (los anteriores a 1985 pueden ser leídos actualmente con aparatos especiales y algunos se han perdido) se conservan en el marco del Proyecto «Vidéo de situations d'enseignement et d'apprentissage» (VISA) de l'Université de Lyon. Pueden ser consultados en el Institut Français de l'Éducation de Lyon (IFE-ENS), en donde se van incorporando progresivamente los videos que son consultados y referenciados por los investigadores que los soliciten, bajo las condiciones de esa institución ${ }^{36}$.

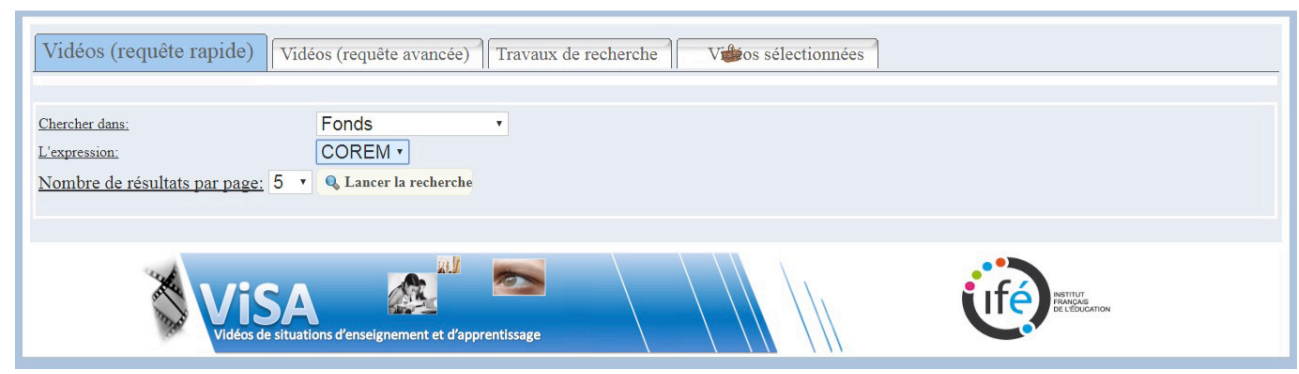

Figura 2. Captura de la pantalla del Proyecto VISA, de acceso a la colección de videos de los fondos COREM

El acceso virtual al CRDM-GB se realiza a través del sitio de la Universidad de Castellón, ${ }^{37}$ la UJI cuyo objetivo es difundir la existencia del Centro de Recursos de Didáctica de las Matemáticas Guy Brousseau (CRDM-Guy Brousseau) y los contenidos de la documentación que alberga, así como las distintas posibilidades de utilización por investigadores en DM y por los profesores de Matemáticas.

\footnotetext{
35 Guy Brousseau, "Accès aux DONNEES DU COREM».

36 Véase http://ife.ens-lyon.fr/manifestations/recherche/visa (consultado el 29-11-2018).

37 Véase http:// www.imac.uji.es/CRDM/index.php (consultado el 29-11-2018).
} 


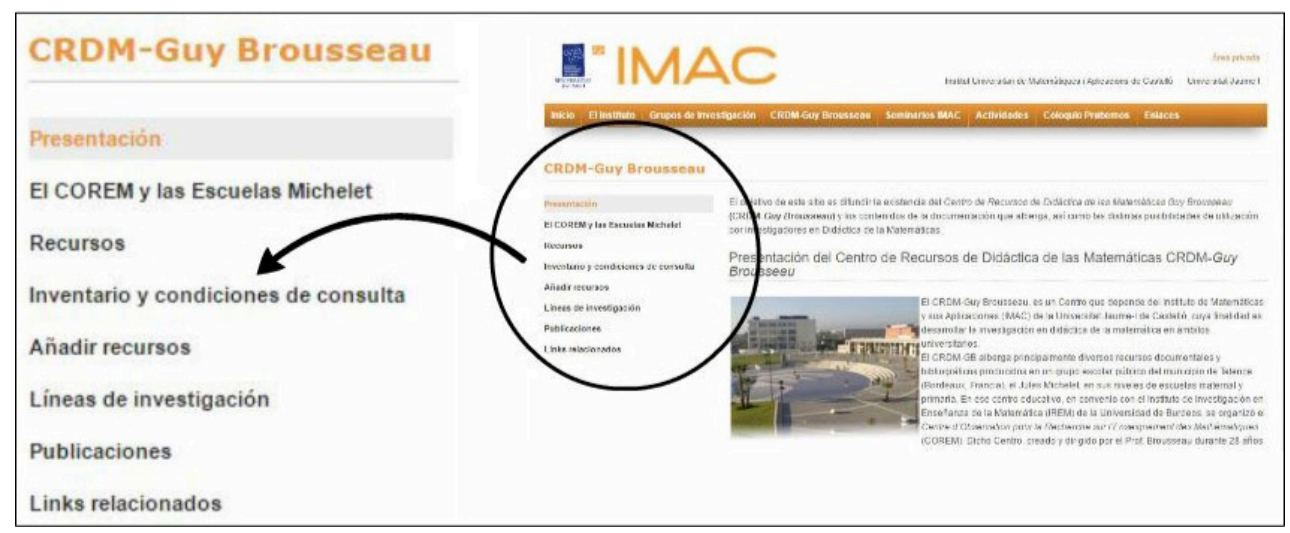

Figura 3. Captura de la pantalla de la web del CRDM en el IMAC.

Entre 1972 y 1999, período en el cual funcionó el COREM, más de 1000 alumnos han concurrido a la escuela J. Michelet y han dejado sus trazas, a través de sus producciones y los saberes desarrollados en esos cursos. Eventualmente es posible seguir el trabajo escolar de algunos de los niños, a lo largo de toda su escolaridad, incluyendo sus desempeños con pruebas de evaluación idénticas (Contrôle d'Acquisition Scolaire y Test d'Acquisition Scolaire) en el transcurso del tiempo, etc. El conjunto de los ejercicios cotidianos efectuados y eventualmente replicados por grupos sucesivos de alumnos, constituye en sí mismo un corpus excepcional.

Finalmente, queremos señalar características de los documentos elaborados: informes, sea para la administración, sea para conservar la traza de lo realizado en los diferentes temas desarrollados en matemática; producciones de alumnos y maestros; registros en video de clases sobre distintos temas matemáticos; tesis de posgrado o artículos de investigación; documentos difundidos por el IREM para la formación inicial o continua de maestros de enseñanza primaria, etc. Consideramos necesario un trabajo de organización, estudio y difusión de esos materiales de DM, y estamos en esa tarea.

\section{NOTAS SOBRE EL DESARROLLO DEL CAMPO EN ESPAÑA Y ARGENTINA}

En la década del ochenta, como muchos otros, iniciamos los primeros contactos personales con la DM atraídas por la visibilidad que los 
IREMs y la ARDM habían dado a la investigación en ese campo. De manera particular, a través de las publicaciones del IREM de Bordeaux.

El nuestro no fue un caso aislado. Francia contó con un número elevado de profesores e investigadores visitantes, entre ellos iberoamericanos, para interactuar con los autores y acceder a las publicaciones, compartir espacios y trabajo con profesores de matemáticas, formadores de profesores, interesados en la investigación en esta nueva perspectiva de investigación, con instituciones francesas de referencia internacional.

El Séminaire National de l'Association de Recherche de Didactique des Mathématiques (ARDM), que celebra varias reuniones anuales generalmente en París, los acogieron y sirvieron a la vez para fomentar las relaciones individuales entre investigadores franceses e iberoamericanos, en este campo de investigación en construcción. El COREM cumplió este papel de «conector» de manera especial, entre formadores de profesores españoles por ser el momento de la creación del área de conocimiento de DM en las universidades españolas y la escasez de doctores, en ese momento.

Tras estos múltiples contactos individuales, la DM empieza su andadura institucional en España, con la creación del Seminario Interuniversitario de Investigación en Didáctica de las Matemáticas (SIIDM) de fecha de constitución el 16 de noviembre de 1991 en la Universidad Complutense de Madrid. Con más de 40 miembros, en su documento de constitución declara:

La Didáctica de las Matemáticas está recibiendo en la actualidad un fuerte impulso como campo de investigación en muchos países, dada la influencia que la formación matemática de los ciudadanos tiene en el progreso científico y tecnológico. Esta investigación se está dirigiendo tanto hacia el nivel técnico-aplicado (tecnología didáctica) como también al nivel del conocimiento científico de carácter básico.

Al tratarse de un campo de conocimiento emergente, las cuestiones de fundamentación teórica y metodológica, y el estudio de las relaciones con otras ciencias, revisten un papel esencial. Por este motivo el Seminario se orienta particularmente hacia estos aspectos. 
Los miembros del Seminario comparten un interés particular por el marco teórico de la Didáctica de las Matemáticas iniciado por el profesor Guy Brousseau, sin que ello suponga el cierre a otras perspectivas que puedan servir de fundamentación para la Didáctica de las Matemáticas. ${ }^{38}$

En 1997 el SIIDM se incorporó como grupo de trabajo de la Sociedad Española de Investigación en EM (SEIEM) en el segundo Simposio que se celebró en Pamplona, al grupo «Didáctica de la Matemática como Disciplina Científica» (DMDC). Entre sus objetivos figura «Potenciar las actividades del Seminario Interuniversitario de Investigación en Didáctica de las Matemáticas —que vienen realizándose desde 1991—y coordinarlas con las de este nuevo Grupo de Trabajo de la SEIEM».39

Queremos resaltar otros dos de los objetivos del grupo, que reflejan la naturaleza diversa de la investigación que realizaban sus miembros en el ámbito de la DM, donde se reconocen los ejes de la didáctica francesa:

Identificar y compartir documentos relevantes sobre los fundamentos teóricos de la didáctica de las matemáticas, teniendo en cuenta las aportaciones de otras disciplinas: matemáticas, epistemología, semiótica, pedagogía, psicología, sociología.

Analizar y confrontar las nociones básicas de aproximaciones epistemológica, antropológica, psicológica y semiótica en didáctica de las matemáticas, en particular las teorías: de situaciones, momentos didácticos, campos conceptuales y funciones semióticas. ${ }^{40}$

Según la información que contamos, en Latinoamérica el proceso de acercamiento ha sido similar. Tras los contactos personales se procede a presentar institucionalmente la DM en diversos países en los primeros

\footnotetext{
${ }^{38} \mathrm{https} / / / w w w . u g r . e s / \sim j g o d i n o / s i i d m /$ welcome.htm(consultada el 19-11-2018)

39 Sociedad Española de Investigación en Educación Matemática, «Didáctica de la Matemática como Disciplina Científica». http://www.seiem.es/grp/dmdc.shtml(consultada el 19-11-2018)

40 Sociedad Española de Investigación en Educación Matemática, «Didáctica de la Matemática como Disciplina Científica».
} 
congresos o reuniones científicas de EM. Un ejemplo, en el Segundo Simposio Internacional en Educación Matemática, en Colombia:

En octubre de 1994, «una empresa docente» realizó en Bogotá, Colombia el Segundo Simposio Internacional en Educación Matemática. A este evento fueron invitadas las profesoras Michèle Artigue (Universidad París VI - IUFM Reims) y Régine Douady (Universidad París VII) de Francia y el profesor Luis Moreno (CINVESTAV) de México. ${ }^{41}$

En Argentina, como en muchos otros países, la conformación de una comunidad de investigación en el campo de la DM se origina en las vinculaciones entre matemáticos y profesores de matemática preocupados por la mejora de la enseñanza, tanto en la escuela secundaria como en la formación de docentes que enseñan matemática. En general las iniciativas tienen lugar en universidades, ${ }^{42}$ y el espacio que tiene mayor antigüedad y sistematicidad está asociado a la Unión Matemática Argentina (UMA). Se trata de la Reunión de Educación Matemática (REM), que se realiza anualmente (desde 1977) asociado al congreso anual de la UMA (cuya primera reunión data de 1951) y al Encuentro de Estudiantes de Matemática (desde 1988). En la REM hay diferentes actividades relativas al estudio de temas específicos de matemática como también de difusión de trabajos de DM desde diferentes perspectivas teóricas. La innovación en la enseñanza tiene allí un lugar de preferencia.

En 1998 se creó la Sociedad Argentina de Educación Matemática (SOAREM), reconocida jurídicamente en el país. Periódicamente esta sociedad realiza un encuentro nacional. ${ }^{43}$

Desde 2006, bianualmente tiene lugar la Reunión Pampeana de Educación Matemática (REPEM) que convoca a investigadores dedicados a estudiar problemas de EM en distintas áreas. Uno de sus objetivos es:

\footnotetext{
41 Cristina Carulla y Pedro Gómez, «Segundo Simposio Internacional en Educación Matemática». http://funes.uniandes.edu.co/317/1/GomezP95-1632.PDF (consultado el 29-11-2018).

42 Una de las excepciones es el Grupo Patagónico de Didáctica de la Matemática, que funciona de manera independiente desde los años 1970. Véase http://gpdmatematica.org.ar/

43 Daniel Pinkasz, Cora Steinberg y Nancy Montes, La Investigación de la enseñanza de Lengua y Literatura y Matemática en la escuela secundaria en Argentina, elaboración del estudio Flavia Terigi, Betina Duarte y Lucía Natale, Primera edición Ciudad Autónoma de Buenos Aires: Facultad Latinoamericana de Ciencias Sociales - FLACSO.
} 
"Ofrecer un ámbito de comunicación, de debate y de reflexión para la comunidad de educadores e investigadores en Educación Matemática». ${ }^{44}$

En 2019 tendrá lugar la novena edición de la Escuela de Didáctica de la Matemática (EDIMAT) que se realiza bianualmente, organizada por la Universidad Nacional de San Martín en colaboración con diferentes universidades del país. Otras universidades nacionales tienen encuentros sistemáticos en los últimos años, entre ellas la Universidad Nacional del Litoral, la Universidad Nacional de Córdoba, la Universidad Nacional de Salta, etc. En muchos de estos encuentros participan investigadores de otros países, y por la temática de este artículo señalamos que los Profesores Brousseau y Chevallard recibieron el diploma de Dr. Honoris Causa por la Universidad Nacional de Córdoba, en 2006 y 2013 respectivamente. Asimismo, en 2013, la Universidad Nacional de Gral. San Martín distinguió con ese diploma a la Prof. Michèle Artigue.

La vinculación de la comunidad argentina de docentes e investigadores en DM con autores vinculados a la didáctica francesa se ve reflejada en diferentes ámbitos. Además de los reconocimientos mencionados, hay numerosas publicaciones en revistas de investigación y en documentos de apoyo curricular que toman como fuentes enfoques teóricos desarrollados por la comunidad francesa. No solamente se toman esos desarrollos teóricos, el panorama es muy amplio y de hecho hay investigadores que mantienen intercambios académicos con docentes/investigadores de otras comunidades, principalmente de la Unión Europea, y países de América.

Esos intercambios también se ponen de manifiesto a través de tesis de posgrado, con diplomas obtenidos en Argentina o en el extranjero.

\section{EL CENTRO DE RECURSOS DE DIDÁCTICA DE LAS MATEMÁTICAS GUY BROUSSEAU (CRDM- GB)}

El CRDM Guy Brousseau, es un Centro que depende de la Universitat Jaume-I de Castellón, España y cuya finalidad es desarrollar la investigación en DM en ámbitos universitarios. La denominación Guy Brousseau para este Centro, «ha sido otorgada por el IMAC en virtud de la relevancia

44 «VII Reunión Pampeana de Educación Matemática». http://repem.exactas.unlpam.edu.ar/ (Consultado el 25-12-2018) 
científica del Prof. Brousseau y de su responsabilidad en la creación y desarrollo del COREM, así como por su implicación en el IMAC, formando parte de su Comité científico, desde el momento de su creación en $2007 »{ }^{45}$

En el año 2010, el Instituto Universitario de Matemáticas y Aplicaciones (IMAC) de la Universidad Jaume I (UJI) de Castellón (España), creó el CRDM-GB para albergar y facilitar el acceso a los recursos documentales y bibliográficos del COREM, cedidos al IMAC. La colaboración incondicional y permanente de un grupo de docentes e investigadores, archivistas e informáticos, está haciendo posible el acceso a los materiales y su difusión vía internet. Uno de los objetivos de este capítulo es dar a conocer la existencia de este Centro virtual para que investigadores debidamente acreditados puedan utilizar estos recursos o incrementarlos, es decir añadir documentos digitalizados o producciones propias que enriquezcan el sitio.46

\section{Recursos que alberga ${ }^{47}$}

Los recursos documentales y bibliográficos que alberga el CRDM son, inicialmente, los producidos por el COREM. Los contenidos matemáticos trabajados, han sido casi todos los del currículo de la enseñanza primaria, ilustrados por situaciones a-didácticas originales y variadas. Contenidos de introducción a la lógica y al razonamiento (designación de objetos, clasificación, orden, etc.); a la enumeración y a los números y sus operaciones elementales; los racionales y decimales: medidas racionales, topología racional y decimal y aproximación, fracciones y homotecias racionales; diferentes medidas (de orden de magnitud diverso); el dominio del espacio y nociones geométricas; etc. Además, fueron objeto de estudio otros temas propios a la teoría de las situaciones: la noción de medio, los diversos tipos de contrato didáctico, la ingeniería y la noción de variable didáctica, etc.

\footnotetext{
${ }^{45}$ IMAC, «Presentación del Centro de Recursos de Didáctica de las Matemáticas CRDM-Guy Brousseau». http://www.imac.uji.es/CRDM/.

46 Dilma Fregona y Pilar Orús, «El Centro de Recursos en Didáctica de la Matemática Guy Brousseau», 114-115.

${ }_{47}$ Pilar Orús y Laura Peydró, «Presentación del Centro de Recursos de Didáctica de las Matemáticas CRDM- Guy Brousseau», XVI Simposio de la Sociedad Española de Investigación en Educación Matemática (SEIEM), 20-22 septiembre, 2012. Universidad Internacional de Andalucía en Baeza (Jaén). http://hdl.handle.net/10234/88849 (consultado el 29-11-2018)
} 
La disponibilidad hoy día de estos recursos de observación, puede contribuir a la formación de nuevas generaciones de investigadores, la profundización en aspectos teóricos y el estudio de lo que concierne a la difusión y enseñanza de las Matemáticas.

El CRDM pone los recursos a disposición de los investigadores interesados, de dos maneras: accediendo a los originales en soporte papel, o a través de los recursos digitales de la Biblioteca de la Universitat Jaume I de Castellón (UJI) en la medida que se vayan digitalizando.

Para la consulta de originales hay que cumplir ciertos requisitos, entre otros respetar: la unicidad y clasificación de dichos recursos; las normativas de protección de datos vigentes en la Universidad y las que permitieron su recogida en la escuela Michelet. Además es necesario identificar la investigación marco de la consulta y la institución de referencia del investigador. ${ }^{48}$

El Inventario es un documento on-line que muestra información sobre los recursos que el CRDM-Guy Brousseau alberga, clasificados por diversos criterios: cursos y niveles escolares, por contenidos curriculares matemáticos o por investigaciones en DM.

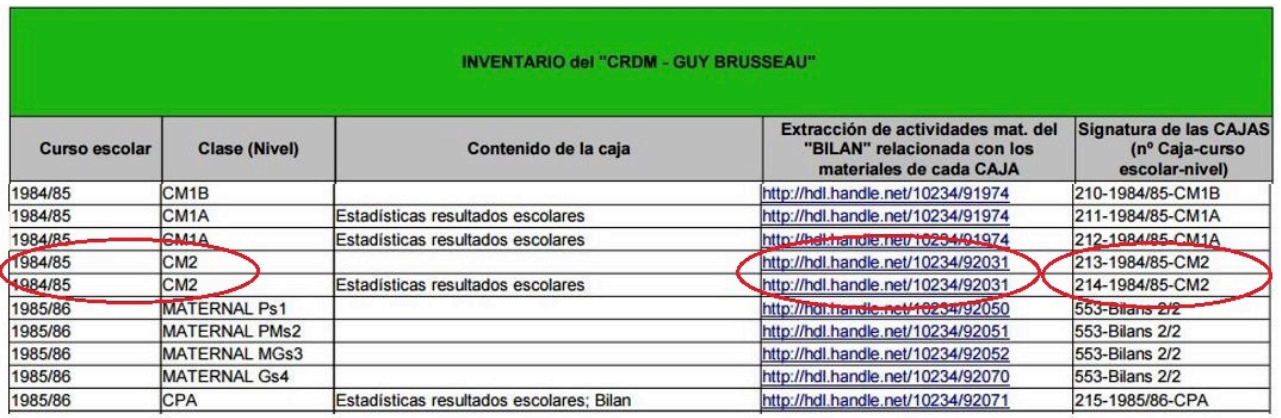

Figura 4. Captura de una pantalla del Inventario de los recursos del CRDM. Seleccionadas las cajas con los recursos de las clases de CM2 del curso 1984/85, identificadas como «cajas 213 y 214 ».

El CRDM-GB pretende ser un centro que vaya incorporando a los recursos iniciales del COREM, cualquier contribución a la DM realizada

\footnotetext{
${ }^{48}$ Véanse condiciones de consulta en: https:/ujiapps.uji.es/ade/rest/storage/PBBX5USXXZF1CPAZUC-
} JF2IT3BQNH55F2 
en el marco de la TSD que sus autores quieran aportar o compartir. ${ }^{49}$ Las producciones de investigación con recursos del CRDM, también se van incorporando, a través de la colección del Repositorio de la Universidad Jaume I. ${ }^{50}$

\section{Nuestro trabajo conjunto a partir de los recursos del CRDM}

A modo de ilustración del trabajo de investigación que realizamos ${ }^{51}$ presentamos un ejemplo sobre la manera que hemos encontrado para producir conocimientos en las condiciones y con los recursos que disponemos.

\section{El problema inicial y las primeras cuestiones}

A demanda de un pequeño grupo de docentes y con la modalidad de taller, en el ámbito de la Universidad Nacional de Córdoba, Argentina, comenzamos a estudiar una secuencia de enseñanza de la división en $\mathrm{N}$ diseñada y llevada a cabo desde la perspectiva de la TSD. Al cabo de un tiempo, pudimos explicitar: «Los propósitos del trabajo que realizamos en el marco del taller son dos: estudiar la secuencia para enseñar la división, y profundizar en el texto para acordar sobre el modo de comunicación de dicha secuencia».52

Si bien nuestro estudio no se enmarca explícitamente en la teoría antropológica de lo didáctico (TAD), hemos tomado algunos aspectos de las dimensiones consideradas por Gascón para analizar un problema didáctico:

La epistemológica, que sitúa a lo matemático en el corazón del problema; la económico-institucional, que despersonaliza la problemática didáctica y delimita la unidad mínima de análisis de los procesos de estudio; y la ecológica, que enfatiza las condiciones necesarias para que sea posible el estudio institucionalizado de las

\footnotetext{
${ }_{49}$ Véase añadir recursos en: http://www.imac.uji.es/CRDM/center.php) (consultado el 29-11-2018)

50 Véase http://repositori.uji.es/xmlui/handle/10234/62668 (consultado el 29-11-2018)

51 Tomado de Dilma Fregona y Pilar Orús, «El Centro de Recursos», 122-127.

52 Dilma Fregona y Pilar Orús, «El Centro de Recursos », 119.
} 
matemáticas y pone de manifiesto las restricciones de todo tipo que inciden sobre dicho estudio. ${ }^{53}$

Hemos tomado aspectos de estas dimensiones, no necesariamente para «el estudio institucionalizado de las matemáticas», sino para el estudio de una secuencia de enseñanza producida en una institución y en un período histórico determinados.

El subtítulo de la publicación estudiada sobre la división es: «Informe de situaciones de enseñanza realizadas con alumnos de tercero, cuarto y quinto grado» ${ }^{54}$. En su redacción, además de los alumnos, participaron los maestros a cargo de esas clases, formadores de maestros e investigadores. Esta distinción es significativa a nivel de las funciones que desempeñaba cada uno en el espacio de un grupo de trabajo colaborativo, que se reunía semanalmente para tomar decisiones sobre el proceso de enseñanza: redacción de planificaciones diarias, discusión y elaboración de proyectos de enseñanza a mediano plazo, preparación de controles y evaluaciones, análisis de clases observadas y de producciones de los alumnos, etc. La publicación refleja en su escritura las discusiones entre maestros, estudiantes de posgrado, profesores formadores e investigadores. Así, la sección dedicada a la presentación de las «actividades» estaba a cargo de los maestros que registraban y redactaban la planificación diaria (previamente), observaban o realizaban esas clases (durante) y recogían producciones de los alumnos (posterior). Las otras secciones ("Antecedentes», «Conclusiones», etc.) habitualmente estaban en manos de otros actores vinculados con ese proyecto de enseñanza.

Decidimos abordar el estudio de esa publicación sin avizorar (tenemos que reconocerlo) la riqueza y complejidad del objeto en cuestión. Desde la TAD podemos describir el proceso al involucrarnos en un tipo de tareas para la cual no conocíamos una técnica que nos permitiera resolverla, es decir que, para nosotros, la tarea era problemática. En esa publicación, como en muchas otras producidas en ese contexto, se produjo un fenómeno de naturalización de «couples tâches/techniques, phénomène qui nous fait vivre comme naturelles — ne supposant donc

\footnotetext{
53 Josep Gascón, «Las tres dimensiones fundamentales de un problema didáctico. El caso del álgebra elemental», Revista Latinoamericana de Investigación en Matemática Educativa 14, no. 2 (2011): 210.

${ }_{54}$ Traducción al castellano en http://repositori.uji.es/xmlui/bitstream/handle/10234/143287/.
} 
aucune technique particulière- les tâches qui s'accomplissent normalement dans une institution».55 En el caso que nos ocupa, la institución incluye al Grupo Escolar Michelet y al COREM, y las técnicas nos remiten a prácticas de enseñanza de la matemática. Así, en algunas secciones de la publicación, interpretamos que, para favorecer la reproductibilidad de la secuencia, se señalan aspectos a tener en cuenta, por ejemplo: «es indispensable que los alumnos tengan un cierto dominio del funcionamiento de la numeración y una práctica "correcta" de la suma, de la multiplicación y de la resta». En el espacio del taller nos preguntamos: ¿cuál es el alcance de esta afirmación? ¿Qué significa práctica «correcta»?

Presencialmente o con recurso a la tecnología, tenemos la oportunidad de analizar diferentes aspectos de la secuencia con docentes e investigadores que participaron en su diseño y ejecución. Es más, en los últimos años en la Escuela Michelet, esa publicación es un material de estudio entre los maestros y directivos, con quienes profundizamos las interpretaciones posibles al texto considerando el fenómeno de naturalización mencionado. Entonces, una pregunta de peso es: ¿cómo utilizar los recursos del CRDM para interpretar y difundir las decisiones consideradas claves en esa secuencia?

En ese proceso de estudio, logramos elaborar algunas producciones propias, presentadas en encuentros académicos y eventualmente difundidas en internet. Véase Anexo de este artículo.

¿Cómo logramos, tal como lo muestran nuestras producciones, explorar los recursos documentales y encontrar aquello que nos permitía avanzar en el estudio?

\section{Una exploración de los recursos del CRDM-GB}

Dado que el año de difusión de la publicación del IREM es 1985, supusimos que los referentes documentales tomados provenían de los años inmediatamente anteriores. Comenzamos así la tarea de exploración del inventario (véase Figura 4) con los datos del curso escolar y las clases, y la consulta de los informes anuales correspondientes (bilan). Consideramos

\footnotetext{
55 Marianna Bosch y Yves Chevallard, «La sensibilité de l'activité mathématique aux ostensifs. Objet d'étude et problématique», Recherches en Didactique des Mathématiques 19, no. 1 (1999): 85.
} 
particularmente: CM2 año 1984-855, ${ }^{56}$ CM1 año 1983-84 y CE2 año 198283. Identificamos así las cajas del archivo que nos interesaban e iniciamos la consulta presencial, tal como lo pudimos organizar a partir de los requisitos de la web del CRDM.

Con las cajas frente a nosotros, y los medios para digitalizar documentos, comenzamos a seleccionar aquellos que intuimos constituirían un aporte a nuestra investigación. Iniciamos así tipos de tareas relativas a la digitalización, organización y difusión de los materiales, tareas que abren un proceso que no tenemos completamente identificado y denominado, a los cuales estamos abocadas en la actualidad. Veamos algunos de los documentos que efectivamente constituyen aportes al proyecto:

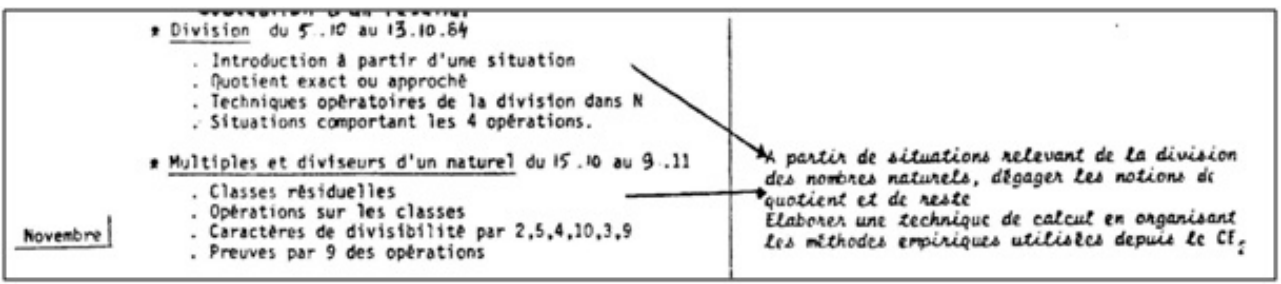

Figura 5. Fragmento del informe digitalizado (bilan) CM2 1984-85.

Una traducción de ese fragmento:

\begin{tabular}{|l|l|}
\hline * División del 5.10 al 13.10 .84 & \\
- Introducción a partir de una situación & \\
- Cociente exacto o aproximado & \\
- Técnicas operatorias de la división en N & $\begin{array}{l}\text { A partir de situaciones que dan } \\
\text { cuenta de la división con números } \\
\text { naturales, obtener las nociones de } \\
\text { cociente y de resto. Elaborar una } \\
\text { técnica de cálculo organizando los } \\
\text { métodos empíricos utilizados } \\
\text { * Múltiplos y divisores de un natural del } 15.10\end{array}$ \\
al 9.11 & \\
- Clases residuales & \\
- Operaciones con las clases & \\
- Priterios de divisibilidad por 2, 5, 4, 10,3,9 9 & \\
\hline
\end{tabular}

Figura 6. Traducción del texto correspondiente a la Figura 5.

\footnotetext{
${ }^{56}$ El año lectivo en Francia inicia en septiembre y cierra en junio del año calendario siguiente.
} 
Algunas preguntas: ¿cuáles fueron las planificaciones de esas clases? ¿Qué problemas se plantean? ¿Con qué estrategias «de base» los van a abordar los alumnos? ¿Se pedirá la explicitación de esas estrategias? ¿Cualquier «método empírico» es aceptado? ¿Qué diferencias hay en la gestión de la clase cuando se trata de explorar los métodos empíricos disponibles o donde hay ciertas institucionalizaciones exigidas? ¿Se pedirá cierta organización en la presentación de las técnicas de resolución? ¿Cuáles son las producciones de los alumnos? ¿Con qué criterios se avanza en actividades constitutivas de la secuencia o se deciden actividades rutinarias?

Buscamos respuestas en las cajas identificadas a través del inventario y encontramos producciones de los alumnos, tanto individuales, como grupales, y fichas didácticas elaboradas por los docentes.

Referido a múltiplos y divisores, observamos una producción individual corregida ( $\mathrm{j}$ : correcto) que da ciertas pistas sobre el modo de estudio. Tal vez sea una evaluación, lo que es claro es que busca seguir individualmente el desempeño de los alumnos en un determinado momento de la secuencia. Ese seguimiento es muy común en las prácticas de enseñanza de la Escuela, ya que son muy frecuentes las actividades grupales.

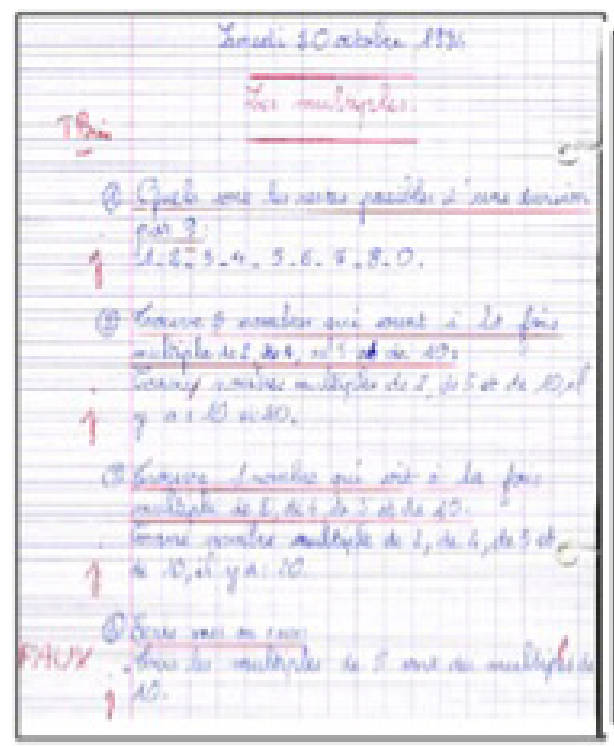

Lunes 20 octubre 1984

Los múltiplos

1. ¿Cuáles son los restos posibles de una división por 9 ?

2. Encuentra dos números que sean a la vez múltiplos de 2, de 5 y de 10.

3. Encuentra un número que sea a la vez múltiplo de 2, 4, 5 y 10.

4. Escribe verdadero of falso:

Todos los múltiplos de 5 son múltiplos de 10.

Figura 7. Producción individual; CM2 1984/85, caja 213, CRDM, UJI. 
Referido específicamente a la enseñanza de la división, la segunda actividad que aparece en el Informe de 1985, plantea el siguiente problema: «Un colocador de baldosas dispone de 2422 baldosas. Debe colocarlas sobre un muro en filas de 23 baldosas. ¿Cuántas filas completas podrá hacer con las 2422 baldosas?».

Como lo expresa la planificación correspondiente, incluida en la publicación, un grupo de alumnos será el de los «colocadores». Disponen de hojas que tienen 2422 cuadrados pero que no tienen 23 cuadrados de ancho, una hoja grande blanca, tijeras, cinta adhesiva y marcadores. La consigna de trabajo para ese grupo es: «Van a representar el muro embaldosado, recortando y pegando las 2422 baldosas colocadas en filas de 23 baldosas. Darán el número de filas completas que hayan podido hacer».

La figura que sigue muestra una fotografía de la producción grupal de los colocadores:

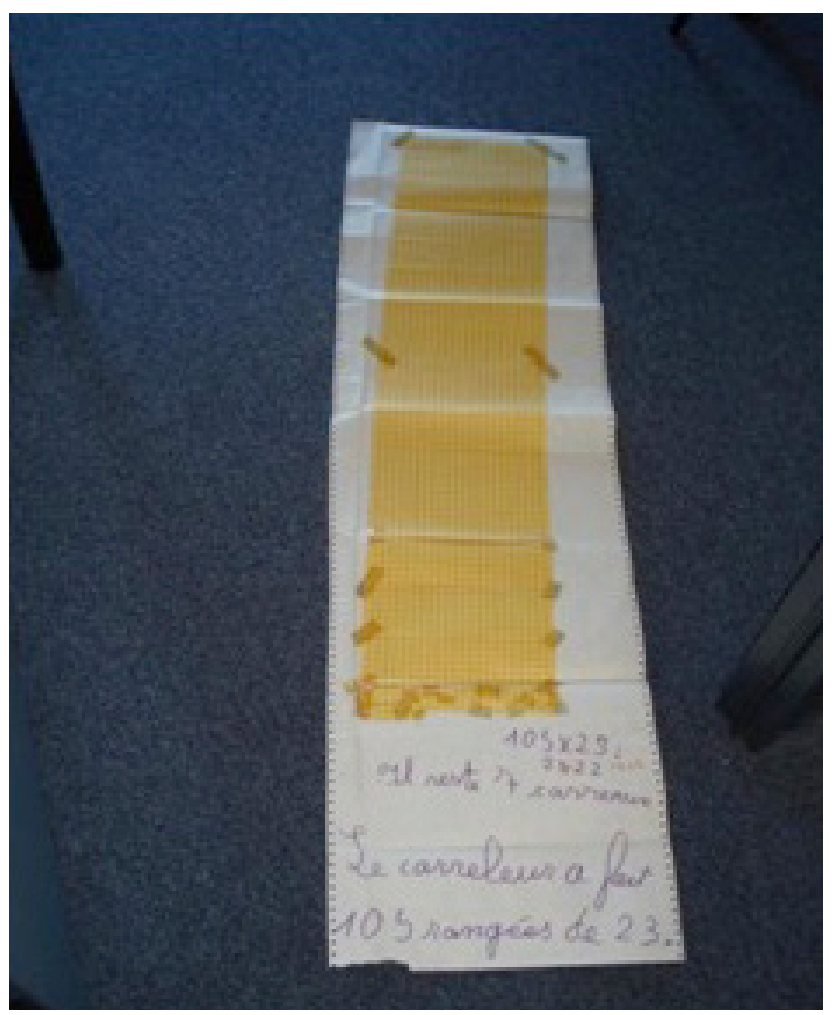

Figura 8. Producción grupal; CM1 1983/84, caja 186, CRDM, UJI. 
La respuesta elaborada por los alumnos es: «105 x $23=2415$ Sobran 7 baldosas. El colocador hace 105 filas de 23». Los otros grupos, abordan el problema haciendo diferentes cálculos.

Sobre la enseñanza de la división, encontramos la ficha didáctica que corresponde a la primera actividad que se plantea a los alumnos de tercer grado, en el octavo mes del curso escolar. El enunciado de ese problema es: "Se quiere distribuir un dulce a cada uno de los 245 niños de una colonia de vacaciones para la merienda. Cada paquete contiene 18 dulces. ¿Cuántos paquetes hay que abrir?».

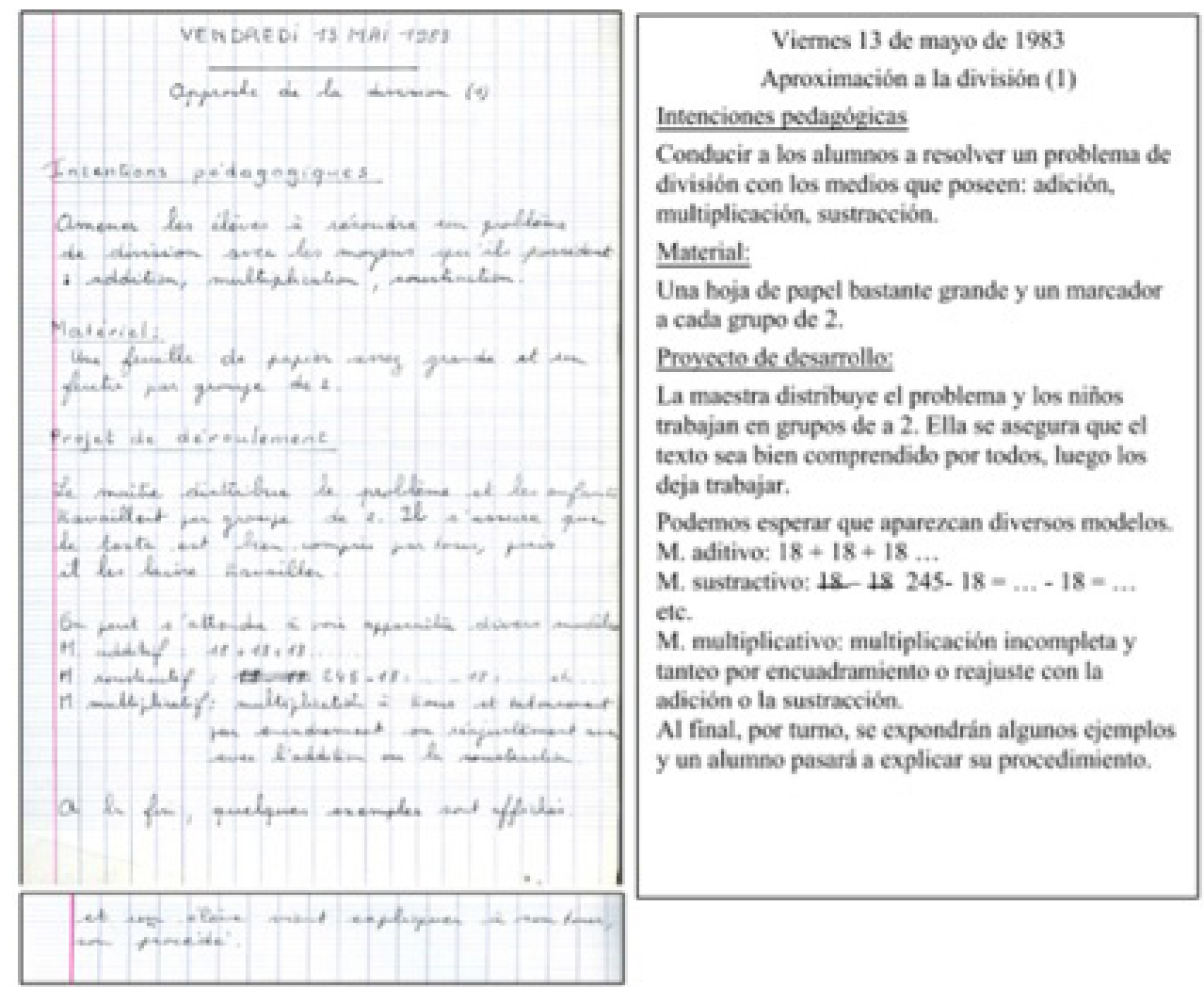

Figura 9. Planificación docente; CE2 1982/83, 13-05-1983, caja 159, CRDM, UJI.

Es importante destacar que en la Escuela Michelet, cuando las secuencias se consideran aceptables se mantienen por varios años los tiempos de estudio de un tema, salvo fenómenos de obsolescencia. Así, en consultas a las docentes sobre decisiones tomadas en las prácticas de 
enseñanza es común que tomen como referencia actividades realizadas en un determinado mes del año lectivo correspondiente, o anterior. Es como si, en mayo, en CE2, con respecto a la división «se hace» tal o cual secuencia de actividades.

\section{Consideraciones sobre nuestro trabajo}

Las experiencias de enseñanza realizadas en el ámbito del COREM, donde los estudios teóricos efectuados en el marco de la TSD se confrontaban con la contingencia en un aula, conllevan también la argumentación de las decisiones fundamentales para conservar el sentido desde la perspectiva teórica adoptada. La recuperación del sentido de dichas experiencias de enseñanza es un objeto de estudio en sí mismo, y a la vez es un insumo para el análisis de toma de decisiones y construcción de modos de resolución de problemáticas en otros escenarios.

La finalidad de la investigación es producir un material de estudio para la formación de docentes que enseñan matemáticas o investigadores en el campo de la DM. Los modos de explorar los recursos documentales albergados en el CRDM-GB son diversos, estamos comunicando en esta sección un modo de hacer, que es el que llevamos a cabo en los últimos años. Contamos además con la permanente y valiosa colaboración de los diferentes actores que diseñaron y realizaron la experimentación en el aula, sus testimonios, las secuencias de lecciones que fueron objeto de investigación y de las lecciones rutinarias que conforman el diseño de enseñanza.

Actualmente, en sucesivas estancias de trabajo en el archivo de la UJI y en el desarrollo de proyectos locales del Seminario Permanente del CRDM-GB ${ }^{57}$ desde el año 2010, hay más de mil documentos digitalizados. Es parte del trabajo compartido en el marco del Convenio entre el IMAC, la FAMAF y la Universidad Nacional del Comahue, organizar esos materiales y ponerlos a disposición del público o con acceso restringido

\footnotetext{
57 Queremos agradecer a Pablo Gregori, profesor del departamento de matemáticas y a Lidón Paris archivista de la UJI, por su generosidad y disposición en el trabajo de digitalización y difusión de los recursos documentales, como miembros del Seminario permanente del CRDM. Un especial agradecimiento además a quienes diseñaron y llevaron a cabo las experiencias de enseñanza y que están siempre disponibles para la consulta: Nadine y Guy Brousseau, Marie-Hélène Salin, Denise Greslard-Nédélec y Christiane Destouesse.
} 
a investigadores debidamente acreditados. Además, estamos trabajando en colaboración con los responsables del proyecto VISA en difundir registros audiovisuales de clases diseñadas y realizadas en el COREM. Hay allí todo un desafío, un camino que ya está iniciado y que empezamos a recorrer.

Otro tipo de producción de los proyectos conjuntos entre FAMAF y el IMAC es poner a disposición la traducción al castellano, con autorización de los autores, de diversos artículos realizados en el marco de las corrientes de investigación francesas (véase Anexo).

\section{A MODO DE CIERRE}

Hemos seleccionado algunos hitos que creemos, a través de datos históricos y de nuestra experiencia, han sido cruciales para el desarrollo de la DM y por sus repercusiones en las comunidades científicas de España y Argentina. Uno de los más importantes, el proyecto y creación del COREM, por parte del IREM de Bordeaux, como centro de observación, para la construcción de una ciencia para la didáctica de las Matemáticas, la Didáctica. Hemos intentado mostrar su importancia a través de los documentos consultados. El sito personal de Brousseau ha sido una constante fuente de referencias, ya que no solamente alberga la mayoría de sus artículos y escritos, sino que también figuran muchas reflexiones posteriores sobre ellos mismos, reformulando, completando y actualizando numerosos conceptos de la TSD. Un ejemplo, los documentos revisados en 2016: Des dispositifs d'apprentissage aux situations didactiques en Mathématiques ${ }^{58}$, Petite histoire du concept "adidactique» ${ }^{59}$, Situation vs tâche, transposition état vs processus ${ }^{60}$, etc. Hemos dejado las citas originales, otras las hemos presentado traducidas, si ya lo estaban en los documentos consultados; por ejemplo, las de nuestros trabajos anteriores.

\footnotetext{
${ }^{58} \mathrm{https} / /$ guy-brousseau.com/2508/des-dispositifs-dapprentissage-aux-situations-didactiques-en-mathematiques-2004/ (consultado el 29-11-2018).

${ }^{59}$ https://guy-brousseau.com/3326/rp-2016-4-petite-histoire-du-concept-adidactique/ (consultado el 29-11-2018).

${ }^{60}$ https://guy-brousseau.com/3263/rp-2016-1-tad-et-tsd/ (consultado el 29-11-2018).
} 
Asimismo, hemos querido remarcar la importancia para la investigación actual en DM de los recursos generados por el COREM, presentando una de las investigaciones conjuntas que hemos realizado a partir de 2012, con recursos del COREM en el marco del CRDM-GB, sobre la enseñanza de diversos contenidos curriculares de enseñanza primaria (división, multiplicación, geometría de figuras planas, etc), así como el trabajo para el mantenimiento y difusión de los recursos.

Invitamos a equipos de investigación a explorar los sitios identificados en este artículo, es posible que entre los documentos encuentren referentes empíricos que les permitan iniciar un proceso de investigación o apoyar un proyecto ya iniciado en torno a una temática específica.

\section{ANEXO}

\section{Traducciones al castellano de diversos artículos franceses de investigación en DM}

Un tipo de producción de los proyectos conjuntos entre FAMAF y el IMAC es realizar y poner a disposición la traducción al castellano, con autorización de los autores, de diversos artículos realizados en el marco de las corrientes de investigación francesas. Esta difusión apunta a ampliar y profundizar la relación de los docentes y formadores de docentes con artículos que son referentes conceptuales en materiales de apoyo curricular, y/o libros o manuales destinados a alumnos de escolaridad obligatoria. En ocasiones, los procesos de divulgación producen distorsiones al sentido de las actividades propuestas, de allí la preocupación por difundir (aunque las traducciones pueden ser mejoradas) en castellano obras que son referentes en el campo. Las traducciones, realizadas por Mabel Aguilar y Dilma Fregona están disponibles en el sitio de la FAMAF: https://www.famaf.unc.edu.ar/la-facultad/institucional/secretar\%C3\%ADas/prosecretar\%C3\%ADa-de-comunicaci\%C3\%B3n-y-divulgaci\%C3\%B3n-cient $\%$ C3\%ADfica/publicaciones/serie-b/

\section{Producciones conjuntas con recursos del CRDM}

Traducciones al castellano y presentaciones a diferentes eventos académicos, están disponibles en la colección «Producciones CRDM_GB» 
del Repositori de la Universitat Jaume I. Véase http://repositori.uji.es/ xmlui/handle/10234/88709

\section{Nota sobre las autoras}

Dilma Fregona es profesora en Matemática, egresada de un instituto superior de formación docente de la ciudad de Rafaela, provincia de Santa $\mathrm{Fe}$, Argentina. Años más tarde obtuvo el diploma de Magíster en Matemática Educativa, de la Sección Matemática Educativa del Centro de Investigación y Estudios Avanzados del Instituto Politécnico Nacional, México. Y luego Doctora en Didáctica de la Matemática por la Universidad de Bordeaux, con tesis dirigida por Guy Brousseau. Entre un diploma y otro pasó alrededor de una década, la formación exigió perseverancia para conseguir las condiciones que la hicieran posible. Los períodos en el extranjero y también de trabajo en universidades públicas fueron posibles por el apoyo de esas universidades nacionales y del Consejo Nacional de Investigaciones Científicas y Técnicas (CONICET). Se desempeñó como docente/investigadora en la Universidad Nacional del Comahue y luego en la Universidad Nacional de Córdoba, actualmente es Profesora Consulta por esta última institución.

Pilar Orús es Licenciada en Ciencias Exactas por la Facultad de Ciencias de la Universidad de Valencia, España. Doctora en Didáctica de la Matemática por la Universidad de Bordeaux (Francia), con DEA y tesis dirigidos por Guy Brousseau, como becaria de investigación del programa bilateral de cooperación, entre los respectivos ministerios de Ciencia e investigación, el MEC (España) y el MRS (Francia). Desde ese momento (1997-1999), es miembro del Séminaire de Didactique des Mathématiques de Bordeaux y del Séminaire National de la Association en Recherches de Didactique des Mathématiques (ARDM) de Francia, realizando frecuentes estancias breves de investigación en Bordeaux y en París. Cofundadora del Seminario Interuniversitario de Investigación en Didáctica de las Matemáticas, actualmente integrado en el grupo DDMC de la Sociedad Española de Investigación en Enseñanza de las Matemáticas. Profesora de Matemáticas y Didáctica de las Matemáticas, en diversos niveles educativos, fundamentalmente (más de quince años) en la Escuela de magisterio de Cheste (Valencia), realizando formación del 
profesorado de Matemáticas y otros tantos años impartiendo Matemáticas Discretas y Álgebra en los estudios de Ingeniería Informática en la Escuela Superior de Ciencia y Tecnología e impartiendo la materia de iniciación a la investigación, del Master de Formación del Profesorado de Secundaria, de la recién creada Universitat Jaume I (UJI), de Castellón. Fundadora del Centro de Recursos de Didáctica de las Matemáticas Guy Brousseau en el Instituto de Matemáticas y sus Aplicaciones de la UJI, actualmente es su Directora honorífica.

\section{REFERENCIAS}

Artigue, Michèle. «Ingénierie didactique». Recherches en didactique des mathématiques 9, no. 3 (1990): 281-308.

Artigue, Michèle. «Ingeniería didáctica». En Ingeniería didáctica en educación matemática. Un esquema para la investigación y la innovación en la enseñanza y el aprendizaje de las matemáticas, editado por Michèle Artigue, Régine Douady, Luis Moreno y Pedro Gómez Guzmán, 33-59. Bogotá: Una Empresa Docente y Grupo Editorial Iberoamérica, 1995.

Ávila, Alicia. (coord.). Rutas de la educación matemática: 30 años de investigación en la revista educación matemática. México: Sociedad Mexicana de Investigación y Divulgación de la Educación Matemática A. C. (SOMIDEM), 2018.

Bosch, Marianna et Yves Chevallard. "La sensibilité de l'activité mathématique aux ostensifs. Objet d'étude et problématique». Recherches en Didactique des Mathématiques 19, no. 1 (1999): 77-123.

Brousseau, Guy. «Processus de mathématisation». La mathématique à l'école élémentaire. Paris: APMEP (1972): 428-457.

Brousseau, Guy. «Peut-on améliorer le calcul des produits des nombres naturels?». Cahier de l'enseignement élémentaire. Bordeaux: IREM de Bordeaux 13 (1978): 195-237.

Brousseau, Guy. «L'observation des activités didactiques». Revue Française de Pédagogie INRP/ENS éditions 45 (1978): 130-140. hal-00515106.

Brousseau, Guy. «L'ingénierie didactique. D'un problème à l'étude a priori d'une situation didactique». En Actes de la 2ème école Ecole d'été de didactique des mathématiques. Olivet: IREM d'Orléans (1982): 39-60.

Brousseau, Guy. «La tour de Babel». Études en Didactique de Mathématiques, Article occasionnel, no. 2. IREM de Bordeaux (1988): 1-17.

Brousseau, Guy. "Utilité et intérêt de la didactique pour un professeur de collège». Petit X 21 (1989): 48-68. 
Brousseau, Guy. ¿¿Qué pueden aportar a los enseñantes los diferentes enfoques de la didáctica de las matemáticas?, Primera parte». Enseñanza de las Ciencias 8, no. 3 (1990): 259-267.

Brousseau, Guy. Iniciación al estudio de la teoría de las situaciones didácticas. Buenos Aires: Libros del Zorzal, 2007.

Chevallard, Yves. La transposition didactique. Du savoir savant au savoir enseigné. Deuxième édition. Grenoble: La Pensée Sauvage, 1991/1985.

Chevallard, Yves. La transposición didáctica. Del saber sabio al saber enseñado. Buenos Aires: Aique, 1997.

Fregona, Dilma y Pilar Orús. La noción de medio en la teoría de las situaciones didácticas. Una herramienta para analizar decisiones en las clases de matemática. Buenos Aires: Libros del Zorzal, 2011.

Fregona, Dilma y Pilar Orús. «El Centro de Recursos en Didáctica de la Matemática Guy Brousseau: un sitio para explorar prácticas de enseñanza de las matemáticas». En Formación de profesores que enseñan matemática y prácticas educativas en diferentes escenarios. Aportes para la Educación Matemática, editado por Dilma Fregona, Silvina Smith, Mónica Villarreal y Fernanda Viola, 109-132. Córdoba (Argentina): FAMAF y Universidad Nacional de Córdoba, 2017.

Gascón, Josep. «Evolución de la didáctica de las matemáticas como disciplina científica». Recherches en Didactique des Mathématiques 52 (1998): 7-33.

Gascón, Josep. «Las tres dimensiones fundamentales de un problema didáctico. El caso del álgebra elemental». Revista Latinoamericana de Investigación en Matemática Educativa 14, no.2 (2011): 203-231.

Groupe de Recherche sur l'Enseignement Elémentaire. Les Observations et les Tables Rondes. Compte rendu du colloque organisé par l'IREM de Bordeaux. Talence: IREM de Bordeaux, 1975, Collection L'analyse de la didactique des mathématiques.

Hernández, Jesús. «Introducción». En La enseñanza de las matemáticas modernas, editado por Jesús Hernández, 11-55. Madrid: Alianza Editorial, 1978.

Kilpatrick, Jeremy. "A History of Research in Mathematics Education». En Handbook for Research on Mathematics Teaching and Learning, editado por Douglas Grouws, 3-38. New York: Macmillan, 1992.

Kilpatrick, Jeremy. «La investigación en educación matemática: su historia y algunos temas de actualidad». En Educación Matemática, editado por Jeremy Kilpatrick, Pedro Gómez y Luis Rico, 1-18. Bogotá: Una empresa docente \& Grupo Editorial Iberoamérica, 1995.

Perrin-Glorian, Marie-Jeanne. «Théorie des situations didactiques: naissance, développement, perspectives». En Vingts ans de didactique des mathématiques en France, editado por Michèle Artigue, Régis Gras, Colette Laborde, Patricia Tavignot, 97-147. Grenoble: La Pensée Sauvage éditions, 1994. 
Pinkasz, Daniel, Cora Steinberg y Nancy Montes. La Investigación de la enseñanza de Lengua y Literatura y Matemática en la escuela secundaria en Argentina, editado por Flavia Terigi, Betina Duarte y Lucía Natale, Primera edición. Ciudad Autónoma de Buenos Aires: Facultad Latinoamericana de Ciencias Sociales - FLACSO 2018.

Rouchier, André. «Naissance et développement de la didactique des mathématiques». En Vingts ans de didactique des mathématiques en France, editado por Michèle Artigue, Régis Gras, Colette Laborde, Patricia Tavignot, 148160. Grenoble: La Pensée Sauvage éditions, 1994.

Vergnaud, Gérard y Catherine Durand. «Structures additives et complexité psychogénétique». Revue Française de Pédagogie 36 (1976): 28-43. 